\title{
'Reparação de lesões ósseas perfurantes produzidas experimentalmente na falange distal de equiinos normais
}

\section{- Repair of experimentally induced perforating bone lesions in the distal phalanx of normal horses}

\author{
Max Gimenez Ribeiro ${ }^{1}$ - CRMV-PRn ${ }^{0} 3418$ \\ * José Luiz de Mello Nicoletti ${ }^{2}$ - CRMV-SP n ${ }^{0} 1081$ \\ Armen Thomassian $^{3}$-CRMV-SP $n^{0} 1113$ \\ Carlos Alberto Hussni ${ }^{4}$ - CRMV-SP n ${ }^{0} 4418$ \\ Ana Liz Garcia Alves ${ }^{4}$ - CRMV-SP n ${ }^{0} 5776$ \\ ${ }^{1}$ Professor Assistente - UNIPAR-Umuarama-PR. Mestre em Cirurgia pela FMVZ -UNESP, Botucatu \\ ${ }^{2}$ Professor Adjunto - Depto. de Cirurgia e Anestesiologia Veterinária - FMVZ, UNESP - Botucatu \\ ${ }^{3}$ Professor Titular - Depto. de Cirurgia e Anestesiologia Veterinária - FMVZ, UNESP-Botucatu \\ ${ }^{4}$ Professor Assistente Doutor - Depto. de Cirurgia e Anestesiologia Veterinária - FMVZ, UNESP-Botucatu
}

* Faculdade de Medicina Veterinária e Zootecnia UNESP - Botucatu Departamento de Cirurgia e Anestesiologia Veterinária CEP 18618-000-Botucatu SP e-mail: nicoletti@fmvz.unesp.br

\section{RESUMO}

O objetivo do presente trabalho foi estudar a evolução clínica, radiológica e anatomopatológica de lesões ósseas perfurantes produzidas experimentalmente na falange distal de eqüinos normais. Foram utilizados 8 eqüinos adultos, de ambos os sexos, isentos de problemas relacionados com os pés, nos quais foram produzidas lesões perfurantes na falange distal através da sola, utilizando-se de uma fresa e broca adaptadas a uma furadeira elétrica (fresamento). Os resultados mostraram que a técnica permitiu um amplo e rápido acesso ao osso podal e a reparação das lesões produzidas ocorreu sem qualquer prejuízo à função locomotora, sugerindo que a mesma poderá ser uma alternativa à curetagem óssea geralmente realizada com rineta e bisturi para tratamento de osteíte distal séptica, queratoma, fratura da falange distal com seqüestro ósseo e drenagem de abscesso subsolar.

Unitermos: eqüino, falange distal, lesão perfurante, cicatrização.

\section{Introdução}

$\underline{0}$ pé do cavalo, permanentemente submetido a intensas forças mecânicas geradas na dinâmica locomotora, é sede da maioria das afecções que resultam em claudicação, destacando-se, entre elas, a contusão na sola. A reação inflamatória decorrente do trauma, dependendo da extensão e tempo de evolução, pode causar a desmineralização da falange distal, definida como osteíte podal. Além disso, também as lesões perfurantes do pé, independente da localização, são potencialmente perigosas quando resultam em infec- 
ção, sendo particularmente de difícil tratamento quando atingem estruturas ósseas ou sinoviais articulares, ou bainha de tendão.

A relutância em invadir cirurgicamente as estruturas profundas do pé, explica, muitas vezes, os insucessos com o tratamento conservativo das lesões internas do casco, especialmente aquelas de natureza infecciosa, causadas por traumas perfurantes palmar ou plantar, cujas seqüelas mais freqüentes são osteíte séptica, fratura ou formação de seqüestro ósseo da falange distal.

Assim sendo, recomenda-se tecnicamente a descompressão local, debridamento e curetagem cirúrgica dos tecidos comprometidos incluindo o osso podal para casos de osteomielite e fratura da falange distal com seqüestro ósseo (BAIRD et al.,1990; BERTONE, 1997; DART e PASCOE, 1988; FIRTH, 1987; JORDAN et al., 1996), osteíte podal séptica com seqüestro ósseo (CAUVIN e MUNROE, 1998; GAHLOT et al., 1990; HONNAS et al., 1994a; KLOHNEN, 1997), abscesso subsolar (HONNAS et al., 1994a; HONNAS, 1997; PARKS, 1997) e queratoma (CHANG e MUNROE, 1997; HONNAS et al., 1994b; HUNT et al., 1995; LINFOORD et al., 1994).

Entretanto, pelo que pudemos investigar, existem proporcionalmente poucos estudos controlados sobre ação, efeitos e evolução pós-operatória da curetagem ou remoção parcial da falange distal em eqüinos (CAUVIN e MUNROE, 1998; GAUGHAN et al., 1989; JORDAN et al., 1996; LLOYD et al., 1988; STASHAK, 1991).

O objetivo deste trabalho foi estudar a reparação cicatricial após a produção experimental de lesões perfurantes na falange distal em eqüinos normais.

\section{Material e Métodos}

Foram utilizados oito eqüinos de descarte, adultos e de ambos os sexos, com cascos isentos de anormalidades. Após aparação dos cascos, e vermifugados, os animais foram divididos em dois grupos (G1 e G2) de quatro animais cada (N1, N2, N3 e N4).

As lesões perfurantes na falange distal foram produzidas em ambos os grupos com uma furadeira elétrica da marca Makita Industrial HP 1500 de $110 \mathrm{v}$, de $1 / 2$ polegada, rotação regulável, à qual foi adaptada uma fresa cônica Marca Drillfeille $\mathrm{N}^{\circ} 2$, com $16 \mathrm{~mm} / 30,5 \mathrm{~mm}$ de diâmetro, conicidade de 20 graus e $5 \mathrm{~cm}$ de comprimento e uma broca aço rápido de $1 / 2$ polegada com $15 \mathrm{~cm}$ de comprimento (Figura1).

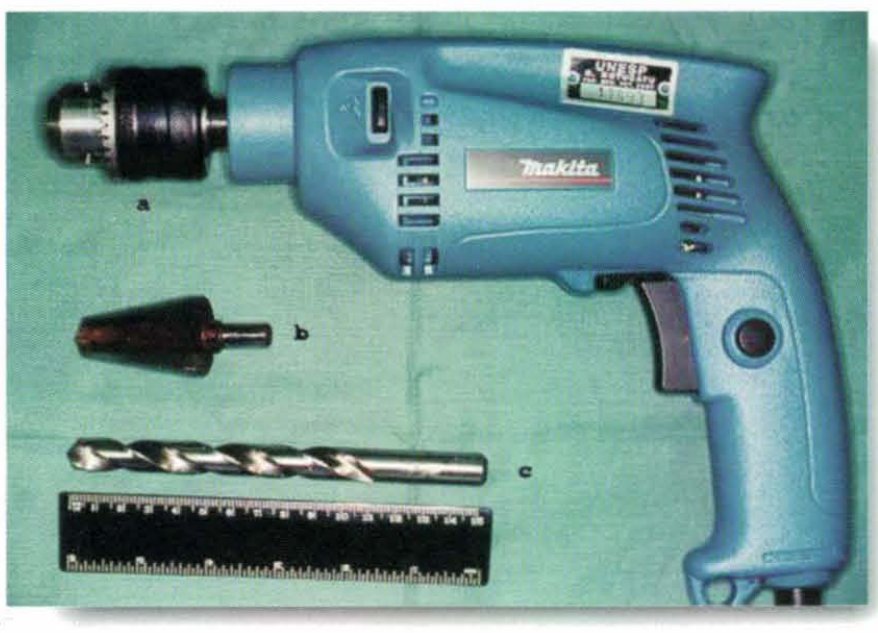

Figura 1 - Furadeira elétrica (a), fresa cônica (b) e broca de aço (c) utilizados para produzir as lesões perfurantes na sola de falage distal.

No Grupo 1, a cirurgia foi realizada no membro anterior esquerdo (MAE) e no Grupo 2, foi realizada no membro anterior direito (MAD).

Precedendo a cirurgia, a falange distal do membro anterior esquerdo (Grupo 1) e direito (Grupo 2) foi radiografada nas posições oblíqua $60^{0}$ dorso-palmar (DP) e látero-medial (LM), sendo denominado momento zero (M0). A técnica utilizada para a projeção oblíqua $60^{\circ} \mathrm{DP}$ foi de $56 \mathrm{kVp}$ e $5 \mathrm{mAs}$ com 0,25 segundos e para a LM, $60 \mathrm{kVp}$ e $8 \mathrm{mAs}$ com 0,25 segundos. O aparelho de Raio $\mathrm{X}$ utilizado foi o modelo portátil FNX 85 com capacidade de $25 \mathrm{~mA}$ e $85 \mathrm{kVp}$.

A medicação pré-anestésica foi feita com romifidina na dose de $100 \mathrm{mcg} / \mathrm{kg}$ IV; indução com éter-gliceril-guaiacol na dose de $100 \mathrm{mg} / \mathrm{kg}$ IV, diluído em $500 \mathrm{ml}$ de soro glicosado $5 \%$ e tiopental sódico na dose de $1,1 \mathrm{mg} / \mathrm{kg}$ e a manutenção em anestesia geral feita com halotano.

A lesão perfurante na falange distal nos animais do Grupo 1 foi realizada após devida limpeza do casco com água e sabão, anti-sepsia com alcool iodado e colocação de torniquete na região metacarpiana, com o animal em decúbito lateral direito com o membro a ser operado para cima.

Inicialmente, foi demarcada com uma rineta uma área na sola com aproximadamente 2 a $4 \mathrm{~cm}$ de diâmetro, imediatamente cranial ao ápice da ranilha do membro anterior esquerdo.

Com a fresa acoplada à furadeira elétrica e girando a baixa rotação, foi realizado na área previamente delimitada um orifício na sola, removendo-se inicialmente o estrato córneo, seguido do pododerme até atingir o ápice da margem solar da falange distal (Figura 2). A seguir, a fresa foi substituída pela broca de $1 / 2$ polegada 


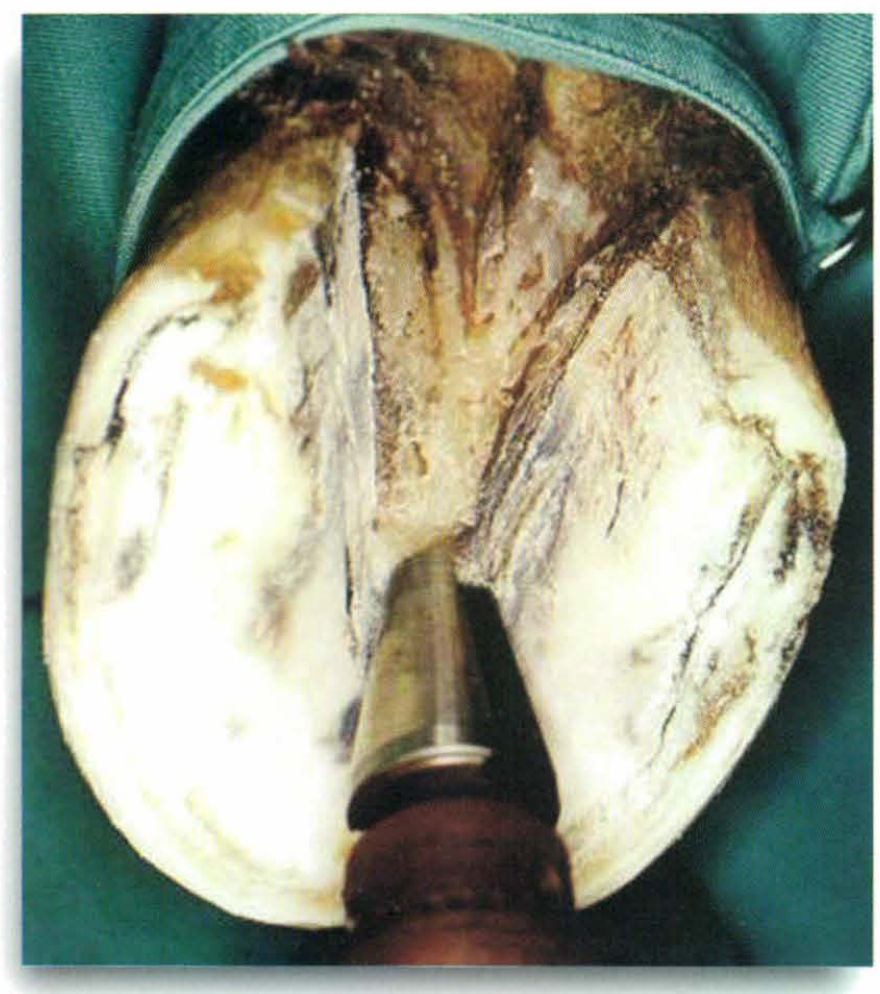

Figura 2 - Delimitação com a fresa cônica da área para produzir a perfuração na sola (queratinizada), pododerme e falange distal.

para atingir e perfurar o osso. Na seqüência, observando-se um ângulo de $45^{\circ}$ em relação à parede dorsal do casco, foi feita a remoção de uma porção da falange distal correspondente ao seu ápice (Figuras $3 \mathrm{a}$ e $3 \mathrm{~b}$ ). Durante a cirurgia, a ferida foi constantemente irrigada com solução salina $0,9 \%$ para remoção dos fragmentos de tecidos resultantes da ação da broca.
Nos animais do Grupo 2, contidos em decúbito lateral esquerdo, a técnica aplicada foi semelhante à do Grupo 1, exceto o local de perfuração da sola e remoção parcial da falange distal, que neste grupo foi feita na porção central do osso podal (Figuras 4a e 4b).

Concluída a intervenção, o orifício produzido na sola foi ocluído com uma mecha de algodão embebida em iodopovidine (PVPI) a $2 \%$ e todo o casco foi envolvido por curativo e protegido por uma bota de couro.

O curativo, inicialmente diário na primeira semana, foi feito com água oxigenada e líquido de Dakin, colocando-se ao final algodão embebido com PVPI a $2 \%$ no interior do orifício. Os curativos seguintes foram feitos a cada 5 dias. Foi aplicado soro antitetânico (5.000 UI) e antiinflamatório não esteróide à base de fenilbutazona na dose de $4.4 \mathrm{mg} / \mathrm{kg}$ IV uma vez ao dia, apenas nos dois primeiros dias de P.O.

\section{Exame Clínico Pós-Operatório}

Foi realizado exame clínico diário nos animais e registrado em protocolo individual as alterações do casco operado e presença de eventuais claudicações, classificadas de 0 a 4, segundo STASHAK (1987).

\section{Exame Radiográfico Pós-Operatório}

Em ambos os grupos foram feitos controles radiográficos simples da falange distal, 24 horas após a cirurgia (momento $1=\mathrm{M} 1$ ) e com a mesma metodologia utilizada no controle pré-operatório. Os exames radiográfi-
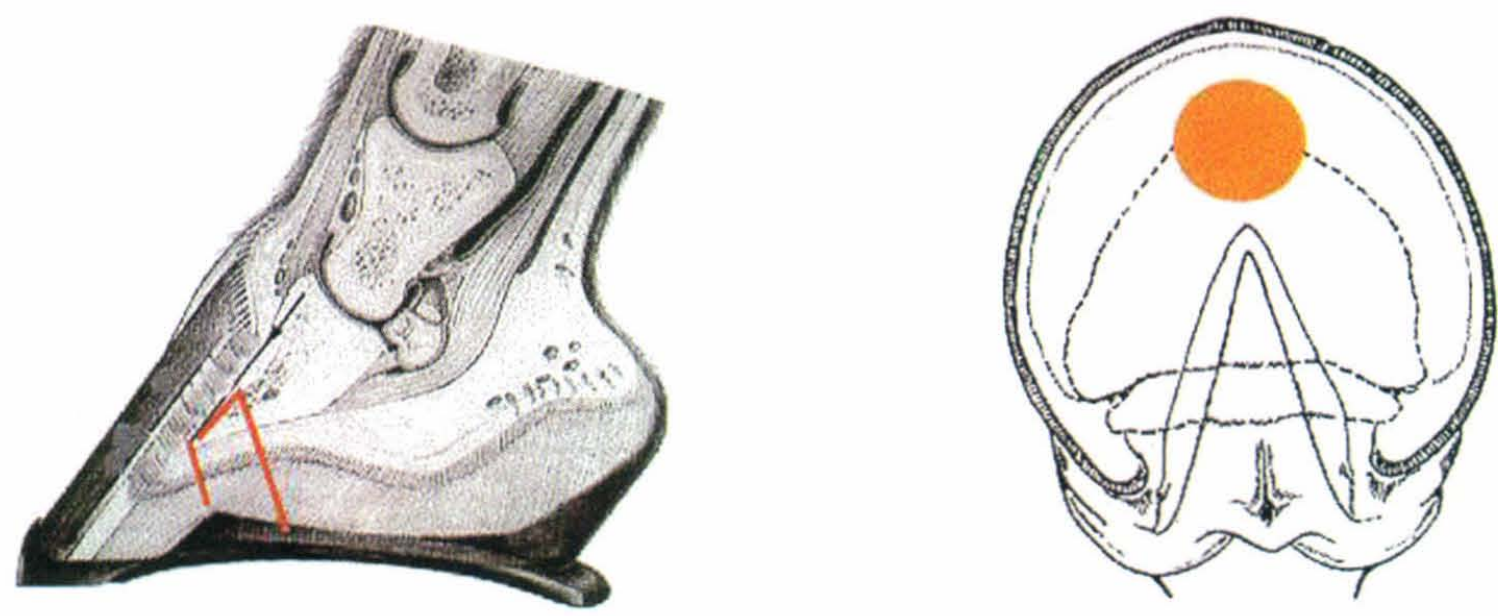

Figura 3a e 3b - Local da lesão perfurante na sola e falange distal produzida nos animais do Grupo 1. 

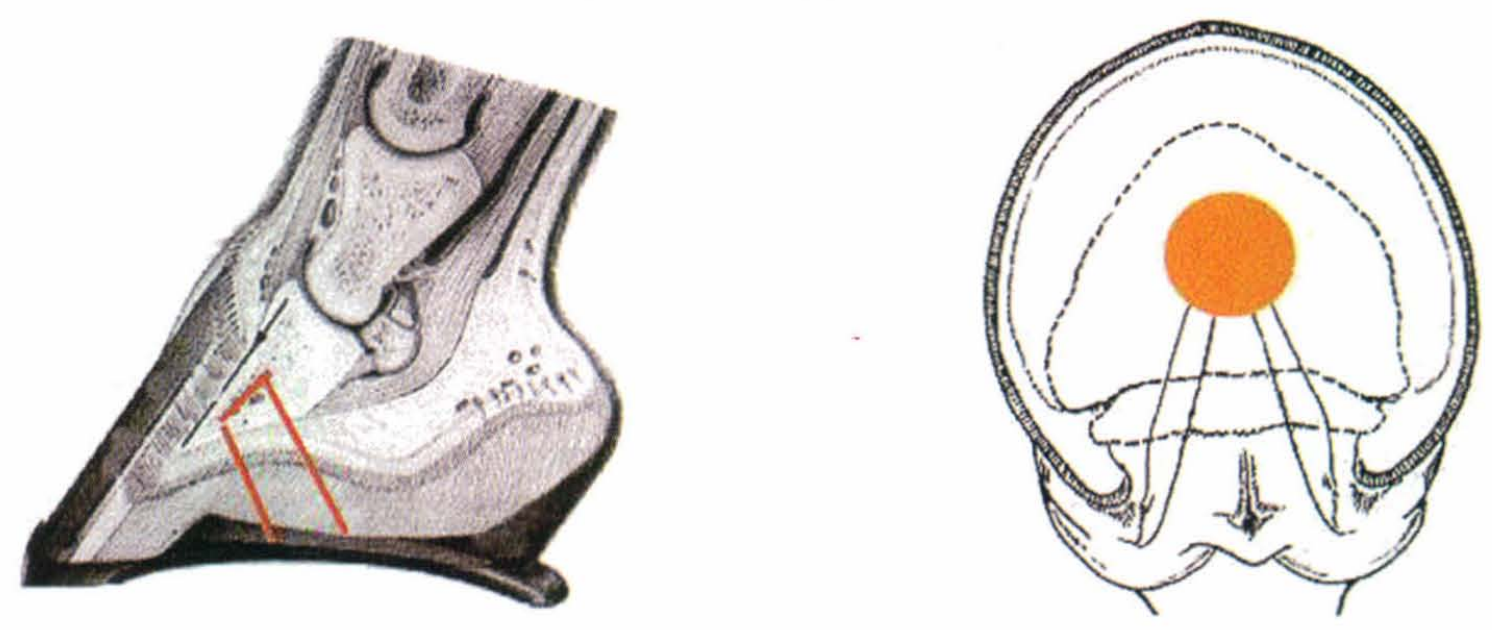

Figura 4a e 4b - Local da lesão perfurante na sola e falange distal, produzida nos animais do Grupo 2.

cos subseqüentes foram realizados a cada 30 dias até o $5^{\circ}$ mês pós-operatório.

\section{Mensuração da lesão produzida na falange distal}

Após a radiografia, realizada no momento 1 (M1) (Figura 5), com auxílio de um planímetro, foi mensurada a lesão produzida no osso. Inicialmente obteve-se uma cópia do contorno da falange distal, tendo como base as radiografias realizadas nos momentos 1 de cada animal, com as devidas lesões. Realizando uma regra de três simples, foi calculada também a percentagem retirada da área plana do osso.

\section{Exames Complementares}

\section{Arteriografia}

Findo o período total de observação pós-operatória, estipulado em 180 dias, foi realizada arteriografia em um animal de cada grupo e as radiografias obtidas em projeção oblíqua $60^{\circ}$ dorso-palmar, com a mesma técnica das radiografias simples.

\section{Exames Anatomopatológicos}

Concluído o período de observação pós-operatória, os animais foram eutanasiados para exames anatomopatológicos, sendo realizados cortes sagitais na região operada tanto para observação macroscópica como para preparação de material para exame histológico com as colorações Hematoxilina-Eosina (HE) e Tricrômio de Masson.

\section{Resultados e Discussão}

A apresentação descritiva dos resultados foi baseada no exame clínico dos animais, quando foram quantificados e classificados os graus de claudicação, observação da cicatrização das feridas produzidas na sola por inspeção direta da lesão, exames complementares com

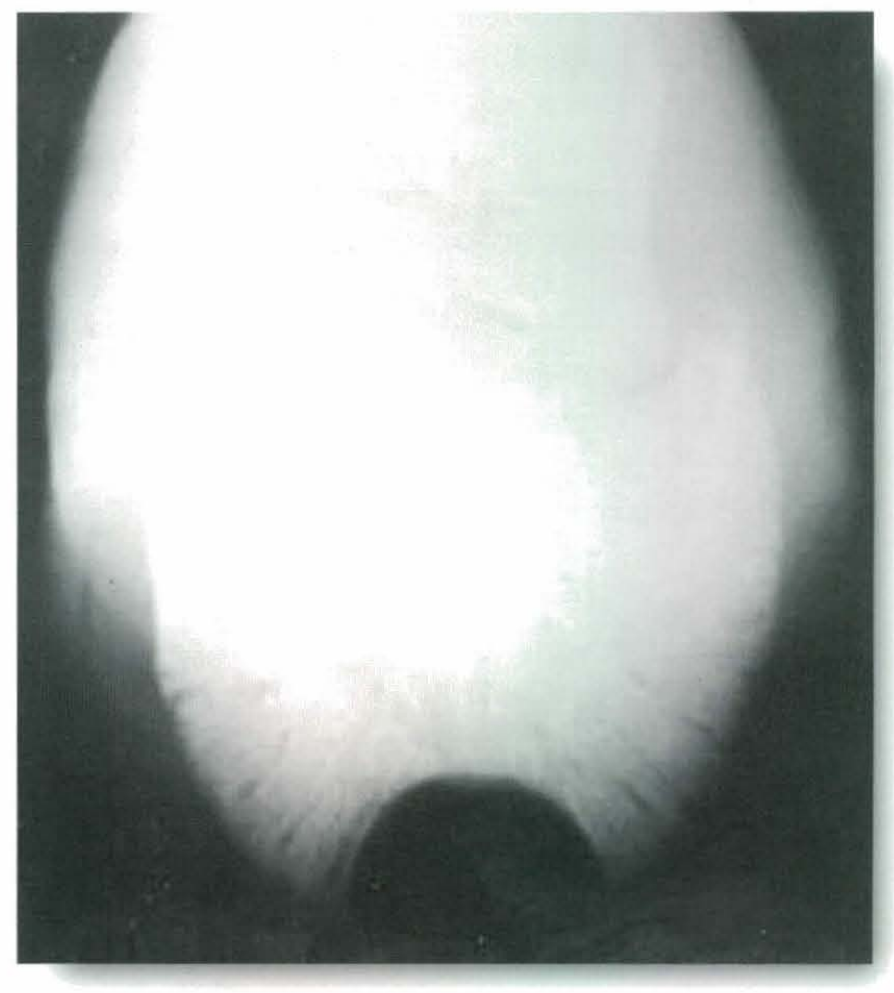

Figura 5 - Radiografia da falange distal no momento 1 (M1 - 24 hs. após a cirurgia) em animal do Grupo 1 e a área óssea removida da margem solar distal. 
radiografias seqüenciais simples, arteriografias e, finalmente exames anatomopatológicos.

Os animais do Grupo 1 apresentaram claudicação de apoio leve (Grau 2) com tendência a melhorar a partir da primeira semana e os do Grupo 2 apresentaram claudicação de apoio leve (Grau 2) com tendência a melhorar a partir da segunda semana. Estes resultados surpreenderam dada a intensidade da lesão produzida no osso e sobretudo por tratar-se de animais hígidos. Isso nos permite considerar a hipótese que a claudicação poderá diminuir gradativamente após o trauma cirúrgico inicial, caso a técnica seja aplicada em casos clínicos de animais com lesões sediadas no osso podal.
Na Tabela 1 estão resumidos os resultados obtidos nos animais dos Grupos 1 e 2.

Como sabemos, os problemas podais em eqüinos são freqüentes e muitos de difícil solução, especialmente quando apresentam características infecciosas, pois, a partir de um foco primário, a lesão pode evoluir e comprometer importantes estruturas do pé como o próprio estrato córneo, articulação interfalangeana distal, bursa do navicular e bainha do tendão flexor digital profundo. No caso da falange distal, indica-se a remoção da porção óssea comprometida por curetagem.

Para a osteíte séptica avançada, relatam-se a retirada de 1 a $10 \%$ da área total da falange distal de eqüi-

Tabela 1 - Resumo dos resultados obtidos nos animais dos Grupos 1 e 2 quanto ao grau de claudicação P.O, área plana retirada da falange distal, porcentagem de osso retirado, tempo de cicatrização da ferida e queratinização da sola, características radiográficas, arteriográficas, macroscópicas e histológicas das lesões.

\begin{tabular}{|c|c|c|}
\hline AVALIAÇÃO/GRUPO & GRUPO 1 & GRUPO 2 \\
\hline $\begin{array}{l}\text { Grau de claudicação P.O. } \\
\text { (tendência do grupo) }\end{array}$ & $\begin{array}{c}\text { Claudicação de apoio leve com } \\
\text { tendência a melhorar a partir da } \\
\text { primeira semana }\end{array}$ & $\begin{array}{c}\text { Claudicação de apoio leve com } \\
\text { tendência a melhorar a partir da } \\
\text { segunda semana }\end{array}$ \\
\hline $\begin{array}{l}\text { Área plana retirada da falange } \\
\qquad \text { distal }\left(\mathrm{cm}^{2}\right)\end{array}$ & $\begin{array}{c}1,5 \text { a } 9,5 \\
\text { média }=5,2\end{array}$ & $\begin{array}{c}1,5 \text { a } 2,5 \\
\text { média }=2,12\end{array}$ \\
\hline $\begin{array}{l}\text { Porcentagem de osso retirado } \\
\text { baseado na área plana }\end{array}$ & 4,0 a $17,4 \%$ & 4,8 a $6,0 \%$ \\
\hline $\begin{array}{l}\text { Tempo de cicatrização da ferida } \\
\text { (dias) }\end{array}$ & $\begin{array}{c}38 \text { a } 54 \\
\text { média }=48 \text { dias }\end{array}$ & $\begin{array}{l}32 \text { a } 70 \\
\text { média }=55 \text { dias }\end{array}$ \\
\hline $\begin{array}{l}\text { Tempo de queratinização da sola } \\
\text { (dias) }\end{array}$ & $\begin{array}{c}50 \text { a } 110 \\
\text { média }=81 \text { dias }\end{array}$ & $\begin{array}{c}\quad 67 \text { a } 107 \\
\text { média }=91 \text { dias }\end{array}$ \\
\hline $\begin{array}{c}\text { Característica radiográfica na } \\
\text { área da lesão }\end{array}$ & Esclerose marginal & $\begin{array}{l}\text { Aumento da radiopacidade } \\
\text { presença de canais vasculares }\end{array}$ \\
\hline $\begin{array}{c}\text { Característica arteriográfica na } \\
\text { área da lesão }\end{array}$ & Discreta área de neovascularização & Nítida área de neovascularização \\
\hline $\begin{array}{c}\text { Característica macroscópica } \\
\text { na área da lesão }\end{array}$ & \multicolumn{2}{|c|}{ Presença de tecido conjuntivo fibroso preenchendo a cavidade óssea } \\
\hline $\begin{array}{l}\text { Característica histológica na área } \\
\text { da lesão }\end{array}$ & $\begin{array}{c}\text { Cicatrização em fase muito inicial } \\
\text { com algumas áreas de lise óssea e } \\
\text { grande quantidade de tecido } \\
\text { conjuntivo frouxo }\end{array}$ & $\begin{array}{l}\text { Indícios de ossificação do tecido } \\
\text { conjuntivo }\end{array}$ \\
\hline
\end{tabular}


nos, podendo chegar até $24 \%$ do osso (CAUVIN e MUNROE, 1998; LINFORD et al., 1994; GAUGHAN et al., 1989), enquanto que em um caso de fratura transversa da falange distal, foi retirado $45 \%$ deste osso (KLOHNEN et al., 1997). Em caso extremo, REDDEN, citado por GAUGHAN et al. (1989), em comunicação pessoal, relata a retirada de toda a falange distal em dois potros, que retornaram ao serviço pois ocorreu uma regeneração funcional do osso observada radiograficamente, o que consideramos surpreendente e algo a ser comprovado.

No presente trabalho, após a retirada de 4 a $17 \%$ da falange distal, servimo-nos de um planímetro para identificar a extensão do defeito produzido e, com uma regra de três simples, calculamos a percentagem da área plana retirada. $\mathrm{O}$ resultado obtido $\mathrm{em} \mathrm{cm}^{2}$, corresponde à área plana removida da falange e a partir da área total, foi calculada a percentagem.

NOTT e MARTEL (1981), ao removerem a falange distal de um potro de três anos, relatam a ocorrência de neoformação óssea, embora sem um controle radiográfico seriado; em nosso estudo, radiograficamente ocorreu um aumento da radiopacidade na área da lesão em três animais, sendo esta radiopacidade melhor observada a partir do M4 (90 dias de P.O.) e histologicamente nos animais do Grupo 2, foram observados indícios de ossificação. Entendemos, entretanto, ser necessária nova pesquisa com pós-operatório mais prolongado para comprovar os indícios de ossificação observados em alguns de nossos animais. KLOHNEN et al., (1997), em um caso de fratura da falange distal onde foi retirado $45 \%$ deste osso, observaram que no décimo mês de pós-operatório havia indícios radiográficos de formação óssea a partir dos 55\% restantes da falange distal. Retornamos, todavia, à nossa posição anterior sobre a necessidade de mais estudos sobre a técnica, prognóstico, evolução radiográfica e clínica, bem como a remota possibilidade de remoção total da falange distal em eqüinos.

Uma condição básica a se observar no tratamento dos problemas infecciosos internos do casco é proporcionar uma boa drenagem do foco infeccioso e para isso, é necessário um amplo acesso à lesão, portanto, abrindose a sola. HONNAS et al., (1994a), citam a retirada de 1 a $2 \mathrm{~cm}^{2}$ da sola de animais com osteíte séptica de falange distal, utilizando rineta, bisturi e realizando a remoção óssea por curetagem. Em nosso estudo, as lesões produzidas experimentalmente na sola variaram de lesões circulares de $1,5 \mathrm{~cm}$ de diâmetro a elípticas de $4 \mathrm{~cm}$ x $3,5 \mathrm{~cm}$ e $6 \mathrm{~cm} \times 2 \mathrm{~cm}$, das quais as lesões na falange distal variaram de $1,5 \mathrm{~cm}^{2}$ a $9,5 \mathrm{~cm}^{2}$ de área plana. Ob- servamos um tempo médio de queratinização da sola de 81 dias no Grupo 1 e 79 dias no Grupo 2, sendo que a ferida cirúrgica cicatrizou com 48 dias no Grupo 1 e 52 dias no Grupo 2, ou seja, o tempo necessário para o preenchimento da ferida produzida no osso com tecido de granulação. Isso significa que aproximadamente em 2 meses, a lesão está reparada sem acarretar problemas futuros de locomoção no animal.

A cicatrização das ferida da sola ocorre de maneira similar à cicatrização da pele; entretanto não ocorre a contração da ferida por causa da epiderme adjacente. Observamos neste trabalho que as feridas cirúrgicas tiveram uma evolução muito satisfatória, sem problemas de infecção ou granulação excessiva ao contrario do que é freqüentemente observado em feridas cutâneas , especialmente na região metacarpiana e metatarsiana (PARKS, 1997). Comparado com a maioria dos relatos, o tempo de recuperação dos animais desta pesquisa foi mais curto ou seja, de 48 e 55 dias respectivamente no Grupo 1 e Grupo 2, quando se completou a cicatrização das feridas e os animais foram liberados a pasto e sem bandagem.

O resultado do fresamento experimental da falange distal, testado nesta pesquisa, permite-nos projetar a utilização da técnica para diversas afecções podais como uma alternativa à curetagem e debridamento com bisturi ou rineta, como são citadas atualmente para casos de queratoma por BERTONE (1997); CHANG e MUNROE (1998); HONNAS et al., (1994b); JORDAN et al., (1996); LLOYD et al., (1988), osteíte podal séptica com seqüestro ósseo por CAUVIN e MUNROE (1988); GAHLOT et al., (1990); GAUGHAN et al., (1989); HARRISON, (1994); LINFORT et al., (1994), drenagem de abscesso subsolear por DEBOWES e YOVICH, (1989); HONNAS et al., (1994a); HUNT et al., (1995); NOTT e MARTEL, (1981); STASHAK, (1991); STECKEL e FESSLER, (1983), ou fratura com presença de seqüestro ósseo da falange distal por BAIRD et al., (1990); BERTONE, (1997); FIRTH, (1987); HONNAS et al., (1994a); KLOHNEN et al., (1997), com a vantagem de abreviação de tempo cirúrgico com o uso do fresamento.

Finamente, em relação ao uso de medicação tópica, as opiniões são divergentes. Sabe-se que, de um modo geral, altas concentrações de anti-sépticos são deletérios para a cicatrização das feridas. No presente trabalho, utilizamos o iodo polivinilpirolidona (PVPI) a $2 \%$ e líquido de Dakin para os curativos pós-operatórios em ambos os grupos e as feridas mantiveram-se secas, sem infecção secundária e com cicatrização satisfatória. 


\section{Conclusões}

O estudo clínico, radiológico e anatomopatológico de lesões ósseas perfurantes produzidas na falange distal de eqüinos normais permitiu-nos concluir que:

1. A recuperação clínica do membro operado foi rápida, sendo de uma semana no Grupo 1 e de duas semanas no Grupo 2.

2. A retirada de até $17,4 \%$ da área plana da falange distal não causou seqüelas e impedimento à locomoção normal do animal.

3. O tempo necessário para reparação da lesão produzida na falange distal, foi, em média, 48 dias no Grupo 1 e 55 dias no Grupo 2, e o tempo necessário para queratinização da sola foi, em média, de 81 dias no Grupo 1 e de 91 dias no Grupo 2.
4. Aos 150 dias de P.O., não foram observados sinais radiográficos de ossificação das lesões produzidas na falange distal.

5. A arteriografia realizada aos 180 dias de P.O. revelou uma nítida neovascularização ocorrida nos animais do Grupo 2.

6. Aos 180 dias de P.O. observaram-se histologicamente indícios de ossificação das lesões produzidas no Grupo 2 .

7. O uso da fresa e da broca, acopladas a uma furadeira elétrica, pode ser uma alternativa para o acesso à falange distal e remoção de lesões que comprometam a viabilidade óssea, pois a técnica de execução simples proporciona um acesso amplo, rápido e preciso ao ápice e ao corpo da falange distal.

\section{SUMMARY}

The aim of this study was to investigate the clinical, radiological and anatomopathological evolution of experimentally induced bone perforating lesions in the distal phalanx of normal horses. Eight adult horses of both sexes totally free of feet disorders were used in the trial. Lesions were produced through the sole of the distal phalanx, using a miller and an electric drill. Results demonstrated that the technique used allowed a complete and precise approach to the pedal bone; lesions were repaired without any impairment to locomotion. This technique may be an alternative for the usual bone curettage of septic pedal osteitis, keratoma, distal phalangeal fracture and sequestration and draining of foot abscess.

Key words: equine, distal phalanx, perforating lesions, healing.

\section{REFERÊNCIAS BIBLIOGRÁFICAS}

1 - BAIRD, A.N.; SEAHORN; T.L.; MORRIS, E.L. Equine distal phalangeal sequestration. Veterinary Radiology, v.31, p. 210-3, 1990.

2 - BERTONE, A.L. Standing surgery of the foot. Journal Equine Veterinary Science, v.17, p.81-2, 1997.

3 - CAUVIN, E.R.J., MUNROE, G.A. Septic osteitis of the distal phalanx: findings and surgical treatment in 18 cases. Equine Veterinary Journal, v.30, p. 512-9, 1998.

4 - CHANG, C.C., MUNROE, G.A. Treatment of a keratoma in a Clydesdale horse. Veterinary Record, v.26, p.453-6, 1997.

5 - DART, A.J., PASCOE, R.R. The pathogenesis and treatment of osteomyelitis and laminitis in a stallion after the prolonged topical application of formalin to the distal phalanx. Australian Veterinary Journal, v. 36, p. 185-8, 1988.

6 - DEBOWES, R.M., YOVICH, J.V. Penetrating wounds, abscesses, gravel, and bruising of the equine foot. Veterinary Clinics of North America: Equine Practice, v.5, p.17994, 1989.

7 - FIRTH, E.C. Bone sequestration in horses and cattle. Australian Veterinary Journal, v.64, p.65-9, 1987.

8 - GAHLOT, T.K.; SHARIFI. D.; PATIL, D.B.; PESHIN, P.K.; SINGH, J. Surgical treatment of septic pedal osteitis in a mare a case report. Archives Faculty Veterinary Medicine, v.6, p.156-9, 1990. 
9 - GAUGHAN, E.M.; RENDANO, V.T.; DUCHARME, N.G. Surgical treatment of septic pedal osteitis in horses: Nine cases (1980-1987). Journal of American Veterinary Medical Association, v.195, p.1131-4, 1989.

10 - HARRISON, L. Puncture wounds of the equine foot. In Practice, v.16, p.294-300, 1994.

11 - HONNAS, C.M.; PELOSO, J.G.; CARTER, G.K.; MOYER, W.A. Surgical management of incomplete avulsion of the coronary band and keratoma in horses. Veterinary Medicine, v.89, p.984-8, 1994a.

12 - HONNAS, C.M.; PELOSO, J.G.; CARTER, G.K.; MOYER, W.A. Diagnosing and treating septic conditions of the equine foot. Veterinary Medicine, v. 89, p.1060-71, 1994b.

13 - HONNAS, C.M. Keratomas of equine digit. Equine Veterinary Education, v.9, p.203-7, 1997

14 - HUNT, R.J.; KOBLUK, C.N.; STECKEL, R.R. Disease of the foot. In: KOBLUK,C.N.; AMES, T.R.; GEOR, R.J. The Horse. Philadelphia: W.B. Saunders, 1995. cap. 35, p.659705

15 - JORDAN, P.; UELTSCHI, G.; ADÉ-DAMILANO, M.; LEPAGE, O.M. Kératome, kyste ou abcès chonique du pied? Diagnostic et traitement. Practice Véterinaire Equine, $v$ 28 , p.311-20, 1996.

16 - KLOHNEN, A.; TROSTLE, S.S.; STONE, W.C.; JOHANNINGMEIER, D.; WILSON, D.G.; HENDRICKSON, D.A. Management of transverse fracture in the distal pha- lanx of a horse. Canadian Veterinary Journal, v. 38 ,p. 561-3, 1997.

17 - LINFORD, S.; EMBERTSON,R.; BRAMLAGE, L. Septic osteitis of the third phalanx: a review of 63 cases. ANNUAL CONVENTION OF THE AMERICAN ASSOCIATION OF EQUINE PRACTITIONERS, 40, 1994, Proceedings $\ldots$, 1994. p. 103 .

18 - LLOYD, K.C.K.; PETERSON, P.R.; WHEAT, J.D.; RYAN, A.E.; CLARK, J.H. Keratoma in horses: seven cases ( 19751986). Journal of American Veterinary Medical. Association, v. 163, p 967-70, 1988.

19 - NOTT, P.J., MARTEL, K.S. Removal of the distal phalanx from a three year old Standardbred filly. Australian Veterinary Practice. v.11, 137-9, 1981.

20 - PARKS,H.A. Wounds of equine foot: principles of healing and treatment. Equine Veterinary Education, v.9, p.317-27, 1997.

21 - STASHAK, T.S. Equine wound management. Philadelphia: Lea \& Febiger ,1991, p. 236.

22 - STASHAK, T.S. Adam's lameness in horses. 4 ed. Philadelphia: Lea \& Febiger,1987, p. 544.

23 - STECKEL, R.R., FESSLER. J.F. Surgical management of severe hoof wounds in the horses: a retrospective study of 30 cases. Compendium Continuing Education Practice Veterinary,v.5, p.435-43, 1983.

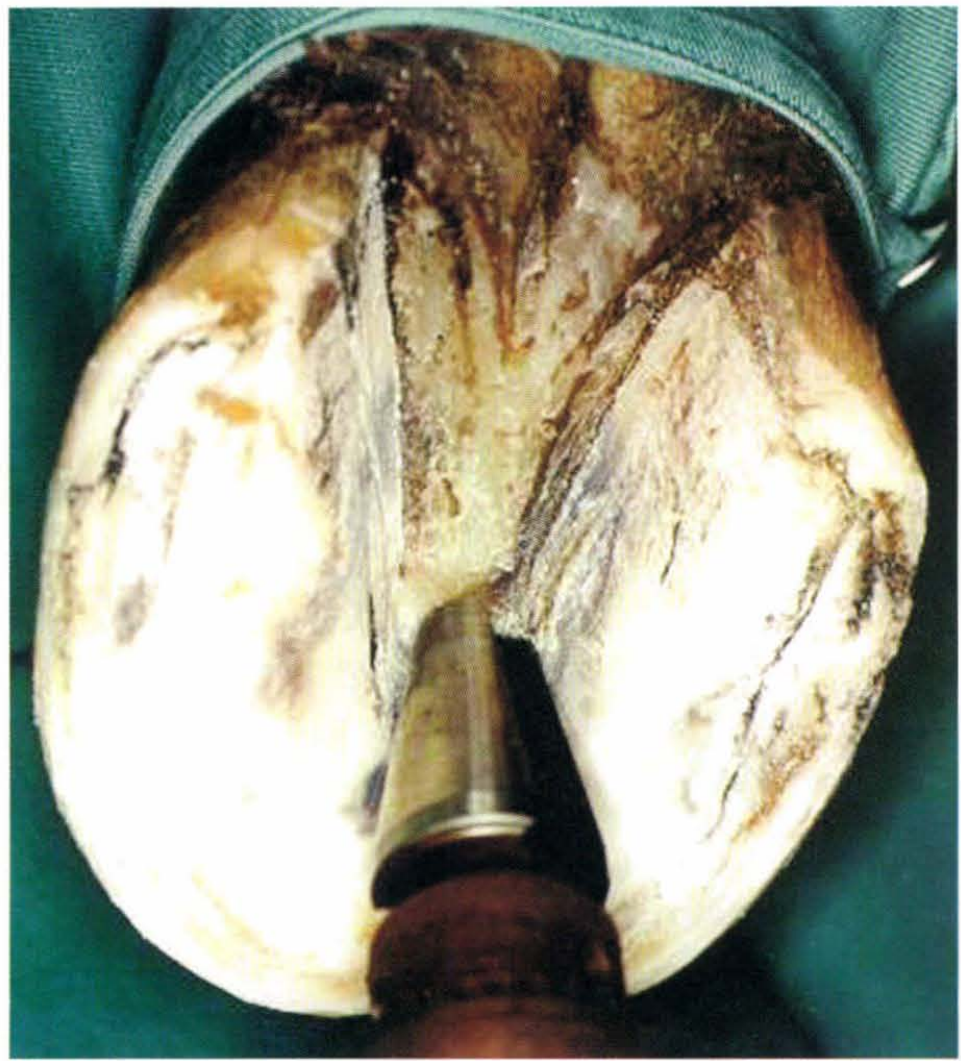

